\title{
Rationale and design of a phase II trial of osimertinib as first-line treatment for elderly patients with epidermal growth factor receptor mutation-positive advanced non-small cell lung cancer (SPIRAL-0 study)
}

\author{
Yusuke Chihara $^{1}$, Tadaaki Yamada ${ }^{1}$, Junji Uchino ${ }^{1}$, Nobuyo Tamiya ${ }^{1}$, Yoshiko Kaneko ${ }^{1}$, Junji Kishimoto ${ }^{2}$, \\ Koichi Takayama ${ }^{1}$ \\ ${ }^{1}$ Department of Pulmonary Medicine, Kyoto Prefectural University of Medicine, Kyoto, Japan; ${ }^{2}$ Department of Research and Development of Next \\ Generation Medicine, Faculty of Medical Sciences, Kyushu University, Fukuoka, Japan \\ Contributions: (I) Conception and design: J Uchino, Y Chihara; (II) Administrative support: None; (III) Provision of study materials or patients: All \\ authors; (IV) Collection and assembly of data: None; (V) Data analysis and interpretation: None; (VI) Manuscript writing: All authors; (VII) Final \\ approval of manuscript: All authors. \\ Correspondence to: Dr. Junji Uchino. Department of Pulmonary Medicine, Kyoto Prefectural University of Medicine, 465 Kajii-cho, Hirokoji-agaru, \\ kawaramachi-dori, Kamigyo-ku, Kyoto, Kyoto 602-0857, Japan. Email: uchino@koto.kpu-m.ac.jp.
}

Background: Osimertinib, a third-generation epidermal growth factor receptor tyrosine kinase inhibitor (EGFR-TKI), has a potent inhibitory effect against both T790M resistance mutations and EGFR-TKI sensitizing in EGFR, with a relatively low affinity for wild-type EGFR. Osimertinib has been approved as a therapeutic agent for patients with T790M-mutation positive advanced non-small cell lung cancer. As a firstline treatment, osimertinib may significantly prolong progression-free survival (PFS) in comparison with the earlier generation first-line standard treatment. Osimertinib has been reported to provide survival benefits to EGFR mutation-positive patients. However, the efficacy and safety of osimertinib as a first-line treatment for patients aged $\geq 75$ years remains to be established.

Methods: In this single arm, prospective, open-label, multicenter, phase II trial, 40 subjects aged $\geq 75$ years with EGFR mutation-positive advanced non-small-cell-lung cancer will be recruited. Patients will be treated with osimertinib $80 \mathrm{mg} /$ day until disease progresses or until the patient meets a discontinuation criterion. The primary endpoint is 1-year PFS. Secondary endpoints are overall response rate, PFS, overall survival, and safety. Thirty-seven patients are required for the present study, as calculated based on normal approximation with a one-sided $\alpha$ level of $5 \%$ and $80 \%$ power, assuming that the expected 1 -year PFS is $70 \%$ and the 1-year PFS threshold is 50\%.

Discussion: We are conducting an intervention study to investigate the safety and efficacy of osimertinib as a first-line treatment agent for EGFR mutation-positive NSCLC in patients aged $\geq 75$ years.

Trial registration number: jRCTs 071180007

Keywords: Osimertinib; elderly patients; progression-free survival (PFS); phase II study

Submitted Sep 15, 2019. Accepted for publication Oct 31, 2019.

doi: $10.21037 /$ tlcr.2019.11.19

View this article at: http://dx.doi.org/10.21037/tlcr.2019.11.19 


\section{Introduction}

Due to population ageing, the incidence of lung cancer is increasing. The highest incidence rates of lung cancer in the US have been observed in the 80-89-year age group in men and $80-84$-year age group in women (1). Moreover, $14 \%$ of lung cancer patients in the US are aged $\geq 80$ years (2). In Japan, 45,000 individuals aged $\geq 75$ years were estimated to have died of lung cancer in 2016 (3). Thus, the development of a treatment strategy for elderly patients with NSCLC is an important issue.

Gefitinib, erlotinib are first-generation, and afatinib is a second-generation epidermal growth factor receptor tyrosine kinase inhibitors (EGFR-TKIs), which have been approved for the treatment of EGFR mutation-positive advanced NSCLC. A meta-analysis of randomized control trials (RCTs) showed that EGFR-TKIs demonstrated a significantly longer median progression-free survival (PFS) than chemotherapy (11 versus 5.6 months) (4). The third-generation EGFR-TKI, osimertinib, has been shown to have a potent inhibitory effect against both sensitizing mutations and resistance by T790M mutations in EGFR, with a relatively low affinity for wild-type EGFR. Osimertinib has been approved for patients with T790M mutation. As a first-line treatment, osimertinib significantly prolonged PFS in comparison with the standard EGFRTKIs in the FLAURA study [18.9 versus 10.2 months; hazard ratio (HR): 0.46] (5).

A phase II trial demonstrated that the efficacy of gefitinib is similar in younger ( $<75$ years) and elderly ( $\geq 75$ years) patients with EGFR-positive patients (6). Osimertinib is well-tolerated compared with firstgeneration EGFR-TKIs (7). The major adverse events observed in the FLAURA study were diarrhea $58 \%$ at all Grades, $2 \%$ at $\geq$ Grade 3 ) and acneiform rash (58\% at all Grades, $1 \%$ at $\geq$ Grade 3) in the osimertinib group, and, in a similar manner, diarrhea $(57 \%$ at all Grades, $2 \%$ at $\geq$ Grade 3$)$ and acneiform rash (78\% at all Grades, $7 \%$ at $\geq$ Grade 3) in the first-generation EGFR-TKIs group. The incidence of adverse events of $\geq$ Grade 3 was reported to be lower in the osimertinib group $(33.7 \%)$ compared with the first-generation EGFR-TKIs group (44.8\%) (5).

Based on these findings, osimertinib is considered to potentially provide benefits to EGFR mutation-positive patients. In Japan, lung cancer clinical practice guidelines define patients aged $\geq 75$ years as 'elderly'; furthermore, based on the results of some clinical studies in Japan, gefitinib and erlotinib are recommended in patients aged $\geq 75$ years $(6,8)$. Although a subset analysis of the data of patients aged $\geq 65$ years was performed in the FLAURA trial, the data obtained for patients aged $\geq 75$ years is highlighted in the elderly patients. In this study, we aim to investigate the efficacy and safety of osimertinib as a firstline treatment for EGFR mutation-positive NSCLC in patients aged $\geq 75$ years.

\section{Methods}

\section{Study design}

This is a single arm, prospective, open-label, multicenter, phase II trial. The present study begun in November 2018 and is currently in progress.

\section{End points}

The primary endpoint is one-year progression-free survival (PFS). Secondary endpoints are response rate (RR), progression-free survival (PFS), overall survival (OS), and safety.

\section{Eligibility criteria}

The inclusion and exclusion criteria are shown in Table 1.

\section{Dose and treatment regimen}

Osimertinib will be administered at a dose of $80 \mathrm{mg}$ orally once daily, at the same time, with or without food. If a scheduled dose is missed, it will be recommended to take osimertinib as soon as possible; however, if it is less than 12 hours until the next scheduled dose, the missed dose of osimertinib will be omitted. Patients will continue to receive oral osimertinib until disease progression or until a discontinuation criterion (Table 2) is met. A schema of the study is shown in Figure 1.

\section{Rationale for the setting of the number of enrolled subjects}

In the FLAURA study, 1-year PFS for EGFR mutationpositive patients treated with osimertinib was approximately $70 \%$ and the median PFS was 18.9 months (5). In addition, the 1-year PFS for EGFR mutation-positive elderly patients who received gefitinib in Japan was reported to be approximately $50 \%$ and the median PFS to be 12.1 months (6). Therefore, 37 patients are required for the present study, 
Table 1 Eligibility criteria

Inclusion criteria

[1] Age $\geq 75$ years at the time of providing informed consent

[2] Histologically or cytologically confirmed non-small cell lung cancer

[3] Patients at a stage of IIIB/IIIC or IV who are inoperable or who experienced postoperative recurrence

[4] EGFR mutation (exon 19 deletion, L858R) known to be associated with EGFR-TKI sensitivity

[5] Treatment-naïve (Preoperative and/or postoperative treatment is permitted)

[6] Patients capable of receiving oral drugs

[7] Patients with at least 1 measurable lesion according to RECIST v1.1 criteria

[8] Eastern Cooperative Oncology Group performance status of 0-2

[9] Patients with normal major organ functions (bone marrow, liver, kidney, etc.) and who satisfy the following criteria in a test conducted within 2 weeks prior to registration (testing on the same day of the week as the day 2 weeks before the registration is permitted)

-White blood cell count $\geq 3,000 / \mathrm{mm}^{3}-\leq 12,000 / \mathrm{mm}^{3}$

- Neutrophil count $\geq 1,500 / \mathrm{mm}^{3}$

- Platelet count $\geq 100,000 / \mathrm{mm}^{3}$

- Hemoglobin $\geq 9.0 \mathrm{~g} / \mathrm{dL}$

- AST $\leq 100 \mathrm{IU} / \mathrm{L}$

- $\mathrm{ALT} \leq 100 \mathrm{IU} / \mathrm{L}$

- Serum bilirubin $\leq 1.5 \mathrm{mg} / \mathrm{dL}$

- Serum creatinine $\leq 2.0 \mathrm{mg} / \mathrm{dL}$

- $\mathrm{SpO}_{2}$ (room air) $\geq 90 \%$

[10] Patients expected to survive for at least 3 months

[11] Patients who have completed a wash-out period for the prior treatment of at least the following duration as of the treatment initiation date (registration is allowed on and after the same day of the week as the day after the following period):

- Chemotherapy (preoperative/postoperative adjuvant chemotherapy): longer than 4 weeks after the last administration date

- Radiotherapy: definitive thoracic radiotherapy: longer than 12 weeks after the last radiation date

- Other radiation therapy: longer than 2 weeks after the last radiation date

Table 1 (continued)
Table 1 (continued)

- Surgery/intervention (inclusive of thoracic drainage): longer than 4 weeks after the last surgery/intervention date

(12) Patients who provided written informed consent by their own free will

Exclusion criteria

[1] Complications of pulmonary disorders such as idiopathic pulmonary fibrosis, interstitial pneumonia, pneumoconiosis, active radiation pneumonitis, drug-induced pneumonia, etc.

[2] Patients on (or unable to discontinue by the time protocol treatment is to start) a CYP3A4 inhibitor treatment (for not less than 1 week) and/or any drugs/herbal supplements that work as an inducer of CYP3A4 (for not less than 3 weeks)

[3] Complications of infectious diseases requiring intravenous administration of antibacterial or antifungal agents

[4] Patients who have any of the following QTC-prolongation risks:

- Mean corrected QT interval at rest of $>470 \mathrm{msec}$ (Fridericia's correction: QTC)

- Clinically important abnormalities (for example, complete left bundle branch block, third-degree heart block, second-degree heart block) in the rhythm, conduction, or waveform of electrocardiogram at rest

- Any factors that increase the risk of QTc prolongation or arrhythmia (for example, cardiac failure, hypokalemia, congenital long QT syndrome, a family history of long QT syndrome or unexplained sudden death in first-degree relatives aged 40 years or younger, or any concomitant drug that is known to prolong QT interval)

[5] Pregnant, lactating, or possibly pregnant women

[6] Active multiple primary cancers (homochronous multiple cancers, or heterochronous multiple cancers with a cancerfree period of not more than 5 years; however, lesions such as carcinoma in situ and intramucosal carcinoma deemed to be cured by local treatment are not included as an active multiple cancer)

[7] Symptomatic brain metastasis

[8] Uncontrolled diabetes

[9] Clinically important complications (such as uncontrolled heart disease, severe arrhythmia in need of medication, persistent watery diarrhea, and so on)

[10] Judged as ineligible to participate in this study by the investigator

ECOG, Eastern Cooperative Oncology Group; EGFR, epidermal growth factor receptor; TKI, tyrosine kinase inhibitor; AST, aspartate amino transferase; ALT, alanine aminotransferase; $\mathrm{SpO}_{2}$, peripheral capillary oxygen saturation. 
Table 2 Discontinuation criteria for treatment protocol

[1] When progressive disease $(\mathrm{PD})$ of the primary disease is observed

[2] When any of the following discontinuation criteria are satisfied due to toxicity:

-When interstitial lung disease (lung fibrosis, pneumonitis) occurs

-When non-hematological toxicity at Grade 4 occurs

[3] When QT interval prolonged accompanied with a symptom/ sign of serious arrhythmia occurs

[4] When the second dose reduction is required

[5] When an adverse event requiring drug interruption for more than three weeks occurs

[6] When a patient wants to withdraw from the protocol treatment

[7] When a patient dies

[8] When it becomes difficult for a patient to continuously receive medical consultations due to his/her move, transfer to another hospital or tight schedule

[9] When any reason arises to exclude the patient from the study following registration

[10] When the investigator decides to exclude the patient for any other reasons

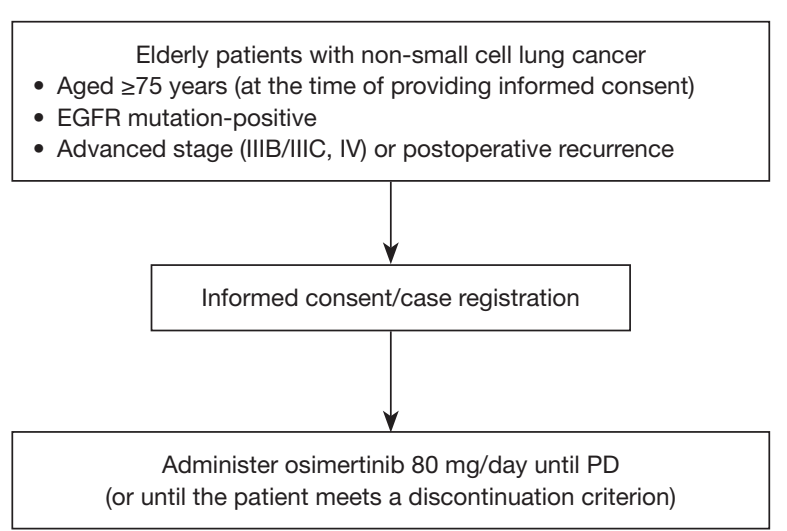

Figure 1 Study profile. EGFR, epidermal growth factor receptor; $\mathrm{PD}$, progressive disease.

when calculated based on normal approximation with a one-sided $\alpha$ level of $5 \%$ and $80 \%$ power, assuming that the expected 1-year PFS is $70 \%$ and the 1-year PFS threshold is $50 \%$. Thus, to account for possible dropouts, the study sample size has been set to 40 .

\section{Population to be analyzed}

Patients enrolled in this study, excluding those with serious protocol violation, will be included in the full analysis set (FAS). The per-protocol set (PPS) will include the remaining subjects from the FAS, after excluding those with violation of inclusion/exclusion criteria or violation for prohibited concomitant drugs/therapies. The safety analysis set (SAF) will include patients who received $\geq 1$ dose of osimertinib.

\section{Statistical analysis}

The 1-year PFS will be estimated by the Kaplan-Meier method, and the confidence interval (CI) calculated using the Greenwood method. If the lower limit of the estimated $90 \%$ CI exceeds the threshold of $50 \%$, it will be considered statistically significant. As for PFS and OS, the KaplanMeier method will be used to estimate the survival curve, median, and annual rate. The Brookmeyer and Crowley method will be used to estimate the median CI, and Greenwood's method will be used to estimate the standard error of the annual rate. The RR and 95\% CI (two-sided) will be estimated via the Wilson method. The severity and incidence of each adverse event will be recorded.

\section{Discussion}

Osimertinib is expected to provide well-tolerated treatment for elderly EGFR mutation-positive patients with fewer serious adverse drug reactions and longer survival benefit compared with first and second generation EGFR-TKIs. To our knowledge, this study is the first prospective trial to investigate the efficacy and safety of osimertinib as a firstline treatment for EGFR mutation-positive lung cancer in patients aged $\geq 75$ years.

\section{Acknowledgments}

We would like to thank Editage (www.editage.jp) for English language editing.

Funding: This work was supported by "Externally sponsored scientific research" in AstraZeneca (ESR-17-13222).

\section{Footnote}

Conflicts of Interest: J Uchino reports grants from Eli Lilly Japan K.K. that are outside of the submitted work. T 
Yamada reports grants from Nippon Boehringer Ingelheim and Ono Pharmaceutical Company that are outside of the submitted work. K Takayama reports grants from ChugaiRoche and Ono Pharmaceutical Company, personal fees from AstraZeneca K.K., Chugai-Roche, MSD-Merck, Eli Lilly, Boehringer-Ingelheim, and Daiichi-Sankyo that are outside of the submitted work. The other authors have no conflicts of interest to declare.

Ethical Statement: The authors are accountable for all aspects of the work in ensuring that questions related to the accuracy or integrity of any part of the work are appropriately investigated and resolved. The study protocol and informed consent documents were approved by the ethical committees of the participating institutions, Clinical Research Network Fukuoka Certified Review Board (No. 18-C06), and informed consent was obtained from all patients.

\section{References}

1. Bravo-Iñiguez C, Perez Martinez M, Armstrong KW, et al. Surgical resection of lung cancer in the elderly. Thorac Surg Clin 2014;24:371-81.

2. Owonikoko TK, Ragin CC, Belani CP, et al. Lung cancer in elderly patients: an analysis of the surveillance, epidemiology, and end results database. J Clin Oncol

Cite this article as: Chihara Y, Yamada T, Uchino J, Tamiya N, Kaneko Y, Kishimoto J, Takayama K. Rationale and design of a phase II trial of osimertinib as first-line treatment for elderly patients with epidermal growth factor receptor mutationpositive advanced non-small cell lung cancer (SPIRAL-0 study). Transl Lung Cancer Res 2019;8(6):1086-1090. doi: 10.21037/ tlcr.2019.11.19
2007;25:5570-7.

3. Center for Cancer Control and Information Services, National Cancer Center, Japan Web site. Available online: http://ganjoho.jp/professional/statistics/statistics.html/ Accessed November 1, 2018

4. Lee CK, Davies L, Wu YL, et al. Gefitinib or erlotinib vs chemotherapy for EGFR mutation-positive lung cancer: Individual patient data meta-analysis of overall survival. J Natl Cancer Inst 2017;109. doi: 10.1093/jnci/djw279.

5. Soria JC, Ohe Y, Vansteenkiste J, et al. Osimertinib in Untreated EGFR-Mutated Advanced Non-Small-Cell Lung Cancer. N Engl J Med 2018;378:113-25.

6. Maemondo M, Minegishi Y, Inoue A, et al. First-line gefitinib in patients aged 75 or older with advanced nonsmall cell lung cancer harboring epidermal growth factor receptor mutations: NEJ 003 study. J Thorac Oncol 2012;7:1417-22.

7. Cross DA, Ashton SE, Ghiorghiu S, et al. OSIMERTINIB, an irreversible EGFR TKI, overcomes T790M-mediated resistance to EGFR inhibitors in lung cancer. Cancer Discov 2014;4:1046-61.

8. Goto K, Nishio M, Yamamoto N, et al. A prospective, phase II, open-label study (JO22903) of first-line erlotinib in Japanese patients with epidermal growth factor receptor (EGFR) mutation-positive advanced non-small-cell lung cancer (NSCLC). Lung Cancer 2013;82:109-14. 